\title{
The Research Review of Public Service Motivation in China
}

\author{
Zheng Xiu Juan \\ School of Public Administration, Yunnan University of Finance and Economics, \\ Yunnan, P. R. China, 650221 \\ ( E-mail: 786564195@qq.eom )
}

\begin{abstract}
The P.S.M is short for public service motivation. As a new research direction in the field of public administration the concept of P.S.M generated the microscopic study of public administration in Chine after 2006. Research on the public service motivation is based on the empirical research of psychological measurement. In China, Empirical research on psychological measurement is included in the western way of thinking. Research and application of the P.S.M theory have positive implication in improving the level of public administration in China. The purpose of this study is to streamline the development and basic research results and to discuss gaps and trend of the research of P.S.M in Chine.
\end{abstract}

Keywords:Public administration, Public service motivation, Summary of the research, China

\section{Introduction}

Compassion which is evaluated from the moral level of civil servants is a recessive psychological process of exciting public servants' altruistic behavior. The dimension designs three questions which include "It is difficult for me to contain my feelings when I see people in distress", "I feel sympathetic to the plight of the underprivileged" and "I am often reminded by daily events how dependent we are on one another". The whole score level of three questions is over 3.9 which is higher compared to other questions. See table 4.

1.2 Research on the public service behavior of civil servants of Chinastarted from ethics it is different study from P.S.M empirical in other countries. The research on public service behavior began in the official virtue ethics of public service personnel research in China. In a study on the role of public service personnel moral believe, Li Jianhua pointed out that the official virtue cannot be equated to professional ethics, professional ethics is just a kind of professional conduct of the sum of specification, and the public service personnel should have moral cognition, according to their own professional public service personnel should not be merely a means to earn a living, in an official on the motivation and the common people no difference. It is not benefit for public service personnel to form the professional role, nor to construct motivation system of serving society for public service personnel. As a public official, his or her value backbone should be serving the people wholeheartedly. And put the interests of the public as the first, honestly and incorruptibly. (Li Jianhua, 2000). Zhang Kangzhi(2003) put forward that social governance behavior model is divided into three patterns that are control, administration and service to advocate moral oriented public administration system should be established, which should be ruling a country by virtue as the main focus, and integrated with ruling by law at the same time, he summarizes the virtue of public service in five aspects: benevolence, justice, realistic, tolerant and temperance.

View of the public sector and civil servants behavior research the characteristic of "economic man". Study is from the macros colic in public paradox Angle in this stage. It is believed that a strong management system is best tool to governing the professional 
conduct of civil servants（Li Chang Wen, 2005）.As the same time bureaucratic corruption became a focus in the study of behavior for the public sector workers. Adopted by the macro perspective, the aim is to inhibit civil servants of deviant behavior.

Toward establishing public servants on a basis of the assumption of economic man researches do not make a clear distinction between the civil servants career motivations and other professional group motivation differences between characters this stage in China.

\section{The Main Reflects of the Research Results from} 2006 to Date

\subsection{Research of the Public Service Motivation Concept}

There are number of Chinese scholars started study of P.S.M, such as Ye Xianbao, Li Xiaohua $(2007,2008)$ , Li Shu (2008) and Zeng Junrong (2008) . They began with study of the concept and connotation, the structure and measurement of the P.S.M. The research have been carried out under the background of the following basic concepts defined as expansion in China.

Public service motivation in essence belongs to an altruistic motive. Public service motivation for altruistic object is the social common people, it is a kind of the altruistic motivation of the universality without discrimination. Public service motivation should be the least affected by object factors of an altruistic motive from the ideal conditions. Perry (1996) defines the "public service motivation" as "personal mainly or entirely based on the motivation of public institutions and organizations driven". Rainey and Stein Burry (Rainey \& Stein Burry, 1999) define public service motivation as serving the community, local, national, or human altruism motivation of interest. Mayo's (Someone, 2004) describes the ideal of public service motivation as some concept that close to love. Sense of public service, strong sense of goal and commitment and dedication are included into this ideal model. Van DE ratio (Vandenabeele, 2007) thinks that public service motivation is some kind of belief, values and attitudes beyond the interests of the individuals and departments. It focuses on the interests of a broader political organization, and motivates people at the right time to take the corresponding actions. Perry and Hondeghem, (Perry \& Hondeghem, 2008) argues that the value orientation of public service motivation may be one person.

Li Ming (2012) proposed P.S.M of cross-cultural research is not enough, He thought that the P.S.M of Asian culture is different from the western P.S.M civil society concept culture. Civil society in China does not yet reach such stage at present, it is not proper to the four dimensions to define and measure of public service motivation.

\subsection{Research on the Effecting Factors of Public Service Motivation}

About effecting factors researches abroad focus on the two angles, antecedents and after factors. Antecedents of the public service motivation formation were discussed, such as family growth environment, education background, and such that how it affects the individuals form public service motivation. The after factor research is mainly on the behavior results that P.S.M public service motivation brings to individuals, such as satisfaction level of the job, influence of organizational commitment, etc. At present most of scholars in the country is using the four dimensions scale of Perry's to carry out empirical study .

There are two purposes of these studies. One is to measure the factors influence of Chinese civil 
servants public service motivation; secondly, to explore how to improve P.S.M of China's civil servants. Ye Xianbao regards demographic variable factors such as the impact of various factors on P.S.M in the correlation from high to low are: work management role, gender, time in position and age. He Put forward there are high correlations between P.S.M and role management. The higher position means the high level P.S.M is and it shows a trend of decreasing as with the lowering of position. And it is concluded that public administration is more suitable for men compared with other social organization( Ye Xianbao,etc.2011).

Zhu Guangnan etc. using the four dimensions scale of Perry's study civil servant's P.S.M in the western China. He concluded that the interests of the public commitment (CPI) were significantly associated with the job involvement. Therefore improving the interests of civil servants commitment is one of the important ways to improve the civil servants job involvement (Zhu Guangnan,2012).

Ye Xianbao etc. think men and women do not have significant differences in the public interest commitments and self-sacrifice dimensions. Women desire to participate in policymaking dimension significantly less than men, women are significantly higher than men in awareness of democratic governance dimensions. Party member status of individuals does not significantly influence the overall level of public service motivation, but for self-sacrifice dimensions has a significant impact; duration in position is a positive correlation factors for public interest commitments and self-sacrifice. But it's a correlation factors for democratic governance dimensions. In public service motivation dimensions, it presents conformity with the functions of service type of dimension, for instance, the
National People's Congress and the Chinese People's Political Consultative Conference in democratic governance dimensions is significantly higher than other departments and administrative departments; while the desire to participate in policy making and public interest commitments dimensions have a higher score. This is different from foreign researches which think P.S.M is the department orientation.

From the Angle of micro management, Li Xiaohua analyses how cognition and personality factors influence the public service motivation in his study. $\mathrm{He}$ used self-developed five scale dimensions including public interest, benefit society, selfrealization, policy making and compassion. He comes to the conclusion: influence of sexfactors in the public service motivation was not significant, age factors only have significant influence on compassion, and sympathy tends to gradually increase with age. With effect of Job roles, apart from public interest dimension, ordinary employees are lower than managers in the rest four dimensions. But it achieves significant difference on empathy dimensions. The compassion level of managers is significantly higher than ordinary employee. $\mathrm{Li}$ Xiaohua thinks that personality factor is a strong forecast quantity of P.S.M, altruism and the five dimensions of public service motivation factors significantly related. Therefore the personality factors of altruism has significantly positive phase impact on P.S.M. In his research, Li Xiao hua works out that role perception is significantly associated with P.S.M, active management role perception can better predict the four dimensions not including self-actualization, and social justice role perception dimension can better predict the benefit of society and compassion. Li Xiaohua in overall concludes that personality and role perception can explain P.S.M in $30.8 \%$ 
variation; organizational factors can explain $30.5 \%$ of the variation of P.S.M separately, in combination of the three factors, it can explain $43.6 \%$ of P.S.M, thus enhanced P.S.M can start from the three aspects( $\mathrm{Li}$ Xiaohua ,2008).

In this research the conclusions were limited at present, no in-depth exploration on how the individuals with other social factors affect the P.S.M, such as background, family environment, etc. Li Xiaohua studied the P.S.M from the perspective of personality. And there is no related research in Chine and abroad. This is a new direction of P.S.M research field in China, which is of innovative significance.

\subsection{Research of public service motivation} dimensions

Structure and dimension of each service motivation is an important aspect of application of P.S.M theory to practice. It is also an important point of this theory to solve cultural differences. Yin Qiang in comparing the various P.S.M dimensions of China to those of American, he points out that these two has significant cultural differences, in the study of China's three dimension of P.S.M aren't stand out except public interests. China's civil servants tend to national consciousness, spiritual needs, social value and the party's vision (Yin Qiang, 2010). Ye Xianbao, etc. after made a measurement of P.S.Mon the public officials to in Fujian province of China, believe that the attraction of public policy and compassion explain $83.164 \%$ of the overall level of P.S.M, fully explains the P.S.M. Zhang tingjun excluding empathy dimensions in Perry scale, put vandenabeele democratic governance dimension in their scale. By this scale study of civil servants P.S.M in Fu Jian province, China "empathy" has not been found, the other three dimensions are verified, and li Ming also draw the same conclusion. Zhang Tinjun think that missing of empathy dimensions in China with the reason that China's civil servants P.S.M is more from the instrumental performance, and it is the professional role norms consciously, rather than personal feelings drive results. (Liu Bangcheng2008 \&Zhang Tingjun,2012 \& li Ming, 2012). Zhu Guangnan etc. study P.S.M of provincial government officials in the central and western China. The conclusion is that it conforms to the Perry 24 items of the scale of the four dimension model. It is obtained that the public interest commitments (CPI) has strongest interpretation for P.S.M, and the attraction of public policy (APM) is the most weak interpretation for P.S.M

Li Xiaohua used a self-designed five dimension scale in his research on P.S.M. of MPA masters in 12 provinces and cities in China. He used self-realization and policy making for rational motivation dimension, public interests and the benefit of society as a specification of P.S.M dimensions, and compassion as a P.S.M emotional dimension 20 items in total. The five factors model contains both contents of the four factor model of western scholars and Chinese cultural background that makes it adapts to the local environment.

Zhu Chunkui study's P.S.Mof civil servants in the Midwest China. He tested the validity of the western P.S.M by integrating descriptive statistics analysis and exploratory factor analysis scale to find out the relationship between the P.S.M dimensions and job satisfaction of China civil servant through correlation analysis and regression analysis. Results show that the P.S.M of civil servants in the central and western area of China, includes five dimensions: selfdedication, mutual willingness and appeal of public policy making, compassion, commitment to public interest (Zhu Chunkui,2011). Li Ming believes that 
the four dimensions of scale that only works in China's social worker recruitment and selection of auxiliary tools can also be used for training and development.

\subsection{Research of public service motivation measure}

P.S.M measurement methods consist of direct measuring method and indirect measuring method. Direct measuring method is using scale of Perry's measurement and evaluation in all levels of public servants. The indirect measuring method is measuring in public service behavior driven (internal requirement), and the public service level of measurement for P.S.M external behavior.

Li Xiao Hua' thinks that indirect measurement is considered alternative measurement methods. The direct measurement is more scientific than the indirect. He studies P.S.M master of public administration of 12 provinces in China from the angle of micro management by a made-up model five questionnaires combined with the personality questionnaire, role cognition questionnaire and group environment questionnaire, he built the first P.S.M research model of the system. This is a prominent contribution to the research in this field in China(Li Xiao Hua,2010).

Zhu Guangnan developed 18 items scale that has higher fitting degree after cut and deletion of the classical 24 P,S.M scale, and on this foundation, he carried out research on the level of P.S.M based on the analysis of Midwest civil servants at the provincial level in China.

Ye Xianbao etc. built the comprehensive scale consists of P.S.M, organizational commitment and job satisfaction measurement in the research process. The role this scale will possibly play is to help improve civil servants job satisfaction and channels for improve P. S. M, and also to help localization of P.S.M scale in China.

The theory of P.S.M using main dilemma in China is the latitude and the establishment of the structure. How to use theory of P.S.M build public management talent selection mechanism, performance, promoting mechanism and P.S.M training mechanism are the main challenge in China. Li Ming thinks there are at least three development spaces for Chine: P.S.M measurement, multiple motives and experiment, implicit measurement and measurement of cultural adaptation in China.

Yang Jiangyun thinks P.S.M measurement research is facing the following difficulties in China: the first thing is measurement tool development; The second step is to performance measure; the third is to find variables measuring and practical ways; The fourth step is to make the tool match with Chinese culture( Yang Jingyun,2012).

\section{Overall Evaluation and Prospect of China's} Public Service Motivation Research

- Nowadays, the description of western's meaning and characteristics of P.S.M concepts are almost accepted in China. But a few numbers of scholars in Chine believe that China's social culture is different from the western civil society culture. Therefore, the concept of P.S.M could not be directly used in China. P.S.M is an altruistic motive, which altruistic behavior has cultural differences. Putting forward concept of Chinese culture background is beneficial to develop our own P.S.M scale and its dimensions, it is necessary for Chine.

- Local P.S.M research in Chinamostly tends to application research and P.S.M mediation variables research under the background of Chinese culture. At present, the research includes P.S.M difference 
ofpublic sector and private sector employees, discussion of results caused by P.S.M and intermediary variables, etc. Such research occupied most of the existing achievements. Meanwhile there are relative differences with conclusions that come from these researches.

P.S.M factors research combined with social demographic factors, organizational environment variables and organizational efficiency creates many different conclusions. More is by using four dimensions of Perry's questionnaire in the study, and a small group of researchers changed or increased Perry's scale dimensions of scale for research. The researches draw different conclusions of factors relevance and contribution and put forward the path for improve and develop China's public servants P.S.M. However the repeat rate is higher with the study conclusions at present, and most of these conclusions are based single research tools and the research area is narrow. Therefore the actual effectiveness of the conclusions is limited.

- The P.S.M dimension research is a very important aspect in China, including dimension and practice of the specific content of the Perry's in China. This area is given priority to empirical research. Through inspection the dimensions of contribution rate $t$ and supplementing the P.S.M dimensions under the background of Chinese culture to measure public servants. Chinese scholars put forward their own points of views, they explain four dimensions in the scale dimension that is missing in use in China. The major conclusion of the P.S.M four dimensions under the background of Chinese culture is insufficient. Some scholars try to build five dimension scale tool under the background of China by delete parts of the four dimension scale dimension, increase the dimension of Chinese culture. Some scholars try to build a more systematic scale by combining P.S.M antecedents and consequences of. This is a new breakthrough in the field of research both in home and at international level.

P.S.M research and development is still immature in China, thus it needs more trials and development to change the reality of research train of thought, and to make the research of P.S.M has a great development in China.

- Perry's scale as the major tool for P.S.M research in China has its limitations. Four dimensional scale is a kind of test methodthat the participant's attitude, with the help of a self-expression. The design of dimensions questions has obvious tendentiousness. The participants may not express their real attitudes and choose the social mainstream orientation answers under Chinese group cultural background. It is adverse in measurement of subject's implicit attitude. Research results may not be realistic. Amongst research results about this point in China at present, only Li Xiaohua measured the PSM by combining the personality factor scale, social cognition with scale P.S.M measurement method after revised the four dimension scale. It is a combination of direct measurement and indirect measurement approach. This is the way to obtain individual recessive manner. Since this tool is complex and massive, it is needed to develop a China localized scale that is simple and easy to practice in China.

\section{Conclusions}

P.S.M as one of human altruism motives, it is an important intermediary factor for improving the efficiency of social management. P.S.M research is even more important for complex social management environment of China nowadays. Focus and purpose of P.S.M research should not stay in the aspect of 
theory, but should be a valuable tool for study in China. This review is far from in-depth and complete for P.S.M research in China. The purpose is to call attentions to P.S.M research in China.

\section{Acknowledgement}

This research was financially supported by the colleague Gan Kaipeng and the classmates of the master of public administration program. They support me to complete the data collection and empirical investigation. With their support, I finished two research papers.

\section{References}

(1) Li, Jianhua. (2000). Official Virtue [D] The Si Chuan People's Publishing House, China, 21-25

(2) Li, Changwen. (2005). Civil Servants "Economic Man" Role And Regulation of Self-interest Behavior Analysis [J] Journal of $\mathrm{Wu} \mathrm{Lu} \mathrm{Mu} \mathrm{Qi}$ Adult Education College,2,58-59

(3) Li, Xiaohua. (2008). Structure and Measure of Public Service Motivation[J]. Journal of Wu Han University, 6, 798-801

(4) Li, Xiaohua. (2010). Public Service Motivation Research, in View of China's MPA Graduate Public Service Motivation of the Empirical Analysis [D] Beijing China Social science press, $177-197$

(5) Li, Ming, \& Ye, Haosheng. (2012). Development and Outlook of Public Service Motivation Measurement [J] Psychological Science, 4, 934
(6) Ma, Chunyan, \&Fan, Shaochen. Research Review of the Theory for Civil Society in Chine http://www.chinaqking.com/yc/2011/18186 8.html

(7) Yin, Qiang. (2010). Evaluation Research in The Theory Of Public Service Motivation-A Technology Institute As an Example In Shanhai [D]. Shang Hai: East China Normail University Pres (Master's papers ), 20-25

(8) Ye, Xian bao, \& Lai, Guimei. (2011). Public Service Motivation: Measurement, Comparison, and Effects, Based on the Analysis of the Sample Data of $\mathrm{Fu}$ Jian Province $[\mathrm{J}]$ Chinese Administrative Management, 8, 108

(9) Yang, Jingyun, \& Zhang, Tingjun. (2012). Public Service Motivation Performance Promotion Theory and Model Building [J] Proceedings of The Cheng $\mathrm{Du}$ School of Administration, 1, 7-11

(10) Zhang, Kangzhi.(2003). Study of Political Civilization and People's Behavior Interaction [J] Teaching and Research , 10, 17-18

(11) Zhu, Chun Kui (2012). The Research Review of P.S.M [J] The Review of The Public Administration 2011,5:147 [12] Zhu GuangNan \& LiMin \&YanMin Civil Servants Public Service Motivation Affect Job Involvement [J] Public Administration Review, 1,124 